\title{
Ultrasonography-guided closed reduction in the treatment of displaced transphyseal fracture of the distal humerus
}

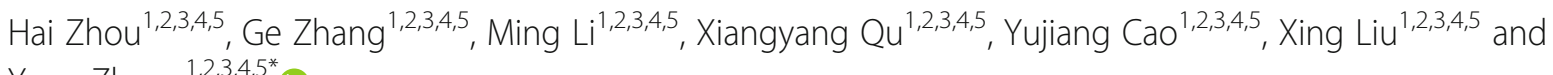
Yuan Zhang ${ }^{1,2,3,4,5^{*}}$ (1)

\begin{abstract}
Background: To evaluate the clinical and radiographic outcomes of ultrasonography-guided closed reduction in the treatment of displaced transphyseal fracture of the distal humerus (TFDH).

Methods: Twenty-seven patients with displaced TFDH were successfully treated by the ultrasonography-guided closed reduction during January 2012 to December 2016 and were retrospectively reviewed. After the mean followup of 34.88 months, the clinical and radiographic outcomes of patients were evaluated. The cubitus varus of the affected elbows was also assessed at the latest follow-up.

Results: The successful rate of ultrasonography-guided closed reduction in the treatment of displaced TFDH was 84\% (27/32). The twenty-seven patients with successful reduction were included for the following analysis. There were 20 males and 7 females included in the study, and the mean age at treatment was $15.39 \pm 3.10$ months; seventeen fractures occurred in the right side elbow and ten in the left side. At the last follow-up, there were significant decreases in the elbow flexion $\left(3^{\circ}, P=0.027\right)$ and range of motion $\left(5^{\circ}, P=0.003\right)$ between the injured and uninjured elbow, respectively, whereas no difference in elbow extension was detected $(P=0.110)$. Flynn's criteria assessment showed that all the patients achieved excellent or good outcomes both in the functional and cosmetic categories. The clinical and radiographic carrying angles at the last follow-up were $11.67 \pm 3.11^{\circ}$ and 10.46 $\pm 3.88^{\circ}$, respectively. And the incidence of cubitus varus after treatment was $7.4 \%$ at the last follow-up.

Conclusion: The ultrasonography-guided closed reduction in the treatment of displaced TFDH is an effective procedure; the adequate fracture reduction can be acquired with the advantages of real-time, non-radioactive, and simple utilization. With the percutaneous pining fixation, satisfactory clinical and radiographic outcomes can be achieved with a low incidence of postoperative cubitus varus.
\end{abstract}

Keywords: Ultrasonography, Closed reduction, Displaced transphyseal fracture of the distal humerus

\footnotetext{
* Correspondence: yuanz2008@126.com

'Department of Orthopaedics, Children's Hospital of Chongqing Medical University, 136 Zhongshan Er Road, Yuzhong District, Chongqing 400014, People's Republic of China

${ }^{2}$ Ministry of Education Key Laboratory of Child, Children's Hospital of

Chongqing Medical University, 136 Zhongshan Er Road, Yuzhong District, Chongqing 400014, People's Republic of China

Full list of author information is available at the end of the article
} 


\section{Introduction}

The transphyseal fracture of the distal humerus (TFDH) is a rare and occurring in young children below age of 3 years old [1]. Owing to the unossification of most epiphyseal centers of elbow at this age, most of the distal humeral epiphysis cannot be visualized directly on radiography, or only the capitellum ossification center can be seen [2, 3]. Nowadays, as the deep understanding of TFDH, it is sufficient to make diagnosis according to the patient's age and the typical radiographic manifestations that posteromedial displacement of proximal radio-ulnar complex is relative to the distal humeral metaphysis [4].

Because of the low incidence of TFDH, many previous studies just reported case reports on this injury with limited impacts on the treatment of TFDH. Recently, some studies reported retrospective studies with small number of consecutive cases $[5,6]$. However, the consensus on the normalized treatment has not been reached, and it is still a need of further seeking on the most appropriate treatment strategy for the TFDH. The open reduction enabled the fracture reduced under the direct vision that can ensure the approximately anatomic reduction. However, there are some inevitable risks involved, such as wound infections, bleeding, and local scars [7]. Closed reduction can avoid most complications related to the open incision, as the closed reduction and percutaneous pining fixation has been established as the conventional procedure in treating displaced supracondylar fractures [8]. Closed reduction with percutaneous $\mathrm{K}$-wire fixation has also become a widely acceptable technique in the treatment of the TFDH [9]. As the closed reduction needs to be assisted by the X-ray fluoroscopy, a great quantity of $\mathrm{X}$-ray is commonly unavoidable to achieve accurate reduction and realignment, which will be resulting in considerable radiation exposure to both surgical staff and patients. Nevertheless, intraoperative elbow arthrography is necessary when treating TFDH with closed reduction which is an invasive procedure with a risk of infection [10].

The ultrasonography (US) is a non-invasive technique that is well-tolerated by children of all ages. The high-resolution transducer depicts internal musculoskeletal structures well, which are generally sufficient in infants and young children [11]. Some case reports have introduced the utilization of US in diagnosis, treatment, and assessment of the TFDH [12, 13]. To our best knowledge, there is no research on the consecutive cases about the intraoperative US-guided reduction of TFDH. In this work, we describe a procedure which uses intraoperative US to assist closed reduction of displaced TFDH and further evaluate the clinical and radiographic outcomes, retrospectively.

\section{Methods}

\section{Patients selection}

After institutional review board approval by Children's Hospital of Chongqing Medical University, we screened patients with the diagnosis of the TFDH from January 2012 to December 2016 to in our single tertiary medical institution. Our inclusion criteria were (1) patients who were initially diagnosed with transphyseal fracture of the distal humerus (we reviewed all the patients' preoperative radiographic data to identify the diagnosis); (2) patients should have undergone closed reduction guided by US as the first choice; and (3) patients whose radiographic data were followed for at least 24 months. The exclusion criteria were (1) patients who have been diagnosed with TFDH in the database, wherein the diagnosis of TFDH was excluded after a thorough radiographic screen; (2) patients with a duration from injury to treatment of more than 5 days; (3) patients who did not undergo closed reduction as the first treatment choice; (4) patients who underwent reduction without US guidance; (5) patients whose follow-up duration was less than 24 months; (6) patients with incomplete clinical and radiographic data at presentation; and (7) patients whose age at the treatment was more than 3 years old.

\section{Surgical technique}

The procedure was performed under general anesthesia, after reassurance of the fracture displacement of the affected elbow by $\mathrm{C}$-arm fluoroscopy. The high-resolution ultrasonography (SonoSite, 5-10 MHz; Inc., Bothell, WA) was used to guide closed reduction intraoperatively. The transducer was coated with a sterile endoscope cover. And the iodophor was used as an ultrasonic couplant. During reduction manipulation, ultrasound imaging of the distal humerus was performed in two standardized longitudinal sectional planes which were the radial/lateral side and the posterior side. The radial side ultrasonography was used to show the lateral displacement of distal end of the fracture; meanwhile, the posterior ultrasonography was used to show the posterior displacement of the distal end of the fracture. The reduction maneuvers were similar to those used for supracondylar fractures. Briefly, all the patients were in the supine position; we first corrected the lateral displacement by pushing the distal fracture fragment under the gentle traction with elbow in extension. The ultrasonic transducer was placed to the radial side of the elbow to assess the correction of the lateral displacement of the fracture. Next, the elbow was flexed while pushing the olecranon with the thumb to correct the posterior displacement of the distal humeral epiphysis. At the same time, the forearm was pronated or supinated to correct the rotation of the fragment. The ultrasonic transducer was placed to the posterior side of the distal 
humerus to evaluate the posterior displacement of the distal fracture end (Fig. 1). Once the acceptable reduction of fracture had been achieved, the elbow was maintained in the maximum flexion to stabilize the reduction and fixation by two K-wires (1.4 mm-1.6 mm in diameter) through percutaneous pinning with the crossed-pin configuration. And the fracture reduction was further confirmed through radiography, which was manifested as the corrected relationship between the distal humeral and forearm. After assurance of the reduction and stablity, the pins were then bent and cut, the arm was placed in long arm casting, and the child was awoken from anesthesia. The long-arm casting plaster was utilized to assist the immobilization of the fracture till removal of the internal fixation pins when fracture healing was documented on two views in the outpatient clinic, usually at 4-6 weeks postoperatively.

\section{Follow-up}

Elbow plain film was taken postoperatively immediately, at 2, 4, and 6 weeks after operation, respectively. Thereafter, patients were followed at the interval of 3 months till the last follow-up.

\section{Clinical evaluation}

The clinical outcomes of patients at the last follow-up were evaluated using Flynn's criteria. The flexion and extension ranges as well as the carrying angles of both affected and unaffected elbows were assessed with the utilization of a goniometer [14]. The carrying angle defined the angle formed by the long axis of the upper arm and the long axis of the forearm in the frontal plane which measured with the elbow extended and the forearm and hand in full supination.

\section{Radiographic evaluation}

The radiographic outcomes were evaluated on anterioposterior and lateral radiographs of both injured and unaffected elbows at the last follow-up among all the patients. The radiographic carrying angles were measured on the anterioposterior radiographs according to the method reported by Chapleau et al. [14].

\section{Cubitus varus}

Cubitus varus deformity was determined by the clinical and radiographic carrying angles, by comparing the injured side with the unaffected side at the latest follow-up. Cubitus varus deformity was defined as a difference of > $10^{\circ}$ in the clinical or radiographic carrying angle, with the injured side value lower than the unaffected side value.

\section{Statistical analysis}

All variables were analyzed by the SPSS 22.0 statistical software, continuous data were indicated by $X \pm \mathrm{SD}$, and the Student ANOVA analysis was used for the comparison of continuous variables. Chi-square test was used for categorical variables. The level of statistical significance was determined at $P<0.05$.

\section{Results}

\section{Patients demographic data}

After screening, there were thirty-two patients included in the present study; among the 32 included patients,
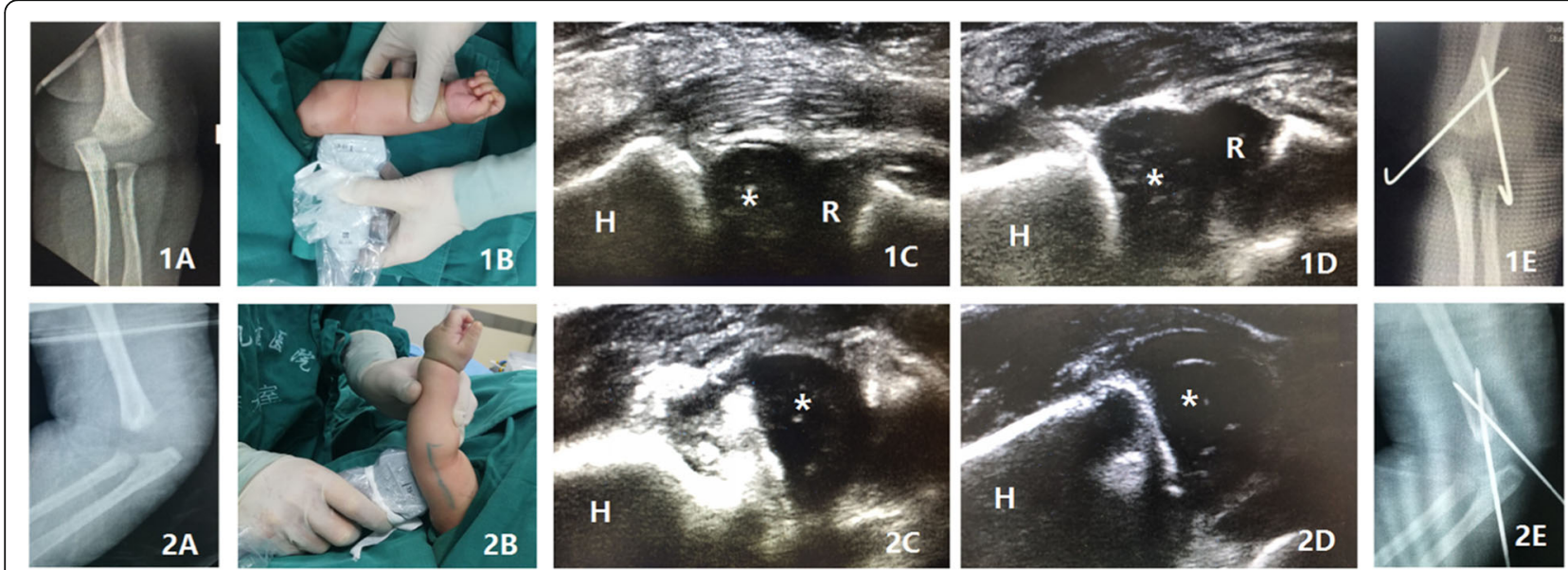

Fig. 1 An 9-month-old boy with TFDH secondary to falling down from a bed. The typical medial displacement of the proximal forearm related to the distal humerus on the anteroposterior radiography (1A). The ultrasonic transducer was placed to the radial side of the elbow to assess the lateral displacement of the fracture (1B). The medial displacement of the humeral capitellum $\left(^{*}\right)$ and the radial head (R) compared with the humerus $(\mathrm{H})(\mathbf{1 C})$. After the closed reduction, the relationship between humeral capitellum $\left(^{*}\right)$ and humerus $(\mathrm{H})$ has been corrected $(\mathbf{1 D})$. The anteroposterior radiography after treatment (1E). The typical posterior displacement of the proximal forearm related to the distal humerus on the lateral radiography (2A). The transducer was placed to the posterior side of the distal humerus to assess the posterior displacement of the distal humerus (2B). The posterior displacement of the humeral capitellum $\left(^{*}\right)$ compared with humerus $(\mathbf{H})(\mathbf{2} \mathbf{C})$. After the closed reduction, the posterior displacement of the humeral capitellum $\left(^{*}\right)$ has been corrected $(\mathbf{2} \mathbf{D})$. The lateral radiography after treatment $(\mathbf{2} \mathbf{E})$ 
there were 27 patients who achieved successful closed reduction under the ultrasound-guided reduction, and 5 patients did not achieve closed reduction and needed further open reduction. The success rate of US-guided closed reduction in the TFDH was $84 \%$ (27/32). We enrolled the 27 patients into further analysis. There were 20 males and 7 females. The average age at treatment was $15.39 \pm 3.10$ months (ranged from 9.40 to 19.43). The average follow-up duration was $34.88 \pm 7.15$ months (ranged from 24.47 to 49.50 ). At the last follow-up, the average age was $50.32 \pm 7.25$ months (ranged from 40.27 to 65.53). The fractures occurred more commonly in the right side $(63 \%, 17$ cases) than that in the left side $(37 \%$, 10 cases). The demographic information on the included patients is available in Table 1.

\section{Clinical outcomes}

At the last follow-up, the average flexion of the injured elbow was $145.26 \pm 4.94^{\circ}$, and the average extension of the injured elbow was $4.57 \pm 4.45^{\circ}$. Differences in elbow flexion were detected between the injured and uninjured side $(P=0.027)$. However, there was no significant difference in the elbow extension between the injured and uninjured side $(P=0.110)$. The mean range of motion was significantly decreased in the injured side when compared to the normal side which was $138.63 \pm 5.95^{\circ}$ and $143.76 \pm$ $5.95^{\circ}$, respectively $(P=0.003)$. The mean clinical carrying angle at the last follow-up was $11.67 \pm 3.11^{\circ}$ for the injured side and $10.63 \pm 7.16^{\circ}$ for the normal side; no difference was detected $(P=0.493)$ (Table 2$)$.

The clinical outcomes were classified as excellent, good, fair, or poor according to the Flynn's criteria. There were two categories of the classification including the loss of motion and the loss of carrying angle in degrees that were compared to the normal elbow. The functional results were excellent in 21 patients $(77.78 \%)$ and good in 6 (22.22\%). The cosmetic results were excellent in 23 patients $(85.19 \%)$ and good in 4 (14.81\%). No patients were noted as the fair or poor grade either in the functional and cosmetic evaluation (Table 3).

Table 1 Demographic data of patients

\begin{tabular}{ll}
\hline Gender $(n)$ & $N=27$ \\
Male & $20(74.07 \%)$ \\
Female & $7(25.93 \%)$ \\
Laterality $(n)$ & \\
Left & $10(37.04 \%)$ \\
Right & $17(62.96)$ \\
The age at injury (months) & $15.39 \pm 3.10(9.40-19.43)$ \\
Average time to surgery (days) & $2.04 \pm 1.13(1-4)$ \\
Follow-up (months) & $34.88 \pm 7.15(24.47-49.50)$ \\
The age at last follow-up (months) & $50.32 \pm 7.25(40.27-65.63)$ \\
\hline
\end{tabular}

\section{Radiographic outcomes}

At the last follow-up, the radiographic carrying angles of both injured elbow and normal elbow were measured. The mean carrying angle was $10.46 \pm 3.88^{\circ}$ in the injured elbow and $10.48 \pm 3.03^{\circ}$ in the normal side, respectively. There were no significant difference between the injured side and the unaffected side $(P=0.752)$ (Table 4).

\section{Cubitus varus}

Two patients $(2 / 27,7.4 \%)$ showed a cubitus varus deformity according to our criteria aforementioned. The two patients demonstrated the deformity in both radiographs and clinical examination.

\section{Discussion}

Ultrasonography is a noninvasive and rapid available technique which is well applied in the diagnosis of musculoskeletal injuries in infants and young children [15]. Supakul et al. [16] suggested that although the posteromedial displacement of the proximal forearm on the radiography is highly suggestive of TFDH, the definite diagnosis can be confirmed with ultrasound. Dias et al. [17] firstly described the ultrasonic diagnosis of TFDH. They demonstrated the ultrasonography characteristics of the distal humerus that the cartilaginous epiphysis is depicted as a hypoechogenic structure with sparkling echoes within it, whereas the cortical bone appears as a highly echogenic structure with posterior acoustic shadowing. In addition, the ultrasonography is a noninvasive examination without ionizing radiations which could obviate elbow arthrography in the detection of TFDH. More importantly, it can also show the direction and extent of fracture displacement which is essential for the guidance of reduction manipulation [18].

A previous study has reported the US could detect cortical discontinuities of $1 \mathrm{~mm}$ or more [19]. Some recent literature also introduced the utilization of ultrasound-guided reduction in forearm and femoral fractures [20-22]. In particular, ultrasonography has the special ability to display the image of the cartilages at the distal part of the humerus in children. Furthermore, intraoperative US-guided reduction provides the image of the fracture displacement continuously in real-time, which help surgeons manipulating the distal segment to reduce without radiation exposure. In the present study, all the fracture reductions were performed under US guidance initially. We achieved a satisfactory successful rate of closed reduction which was $84 \%$ (27/32). Some researchers doubted that US may have limited use because of the requirement of significant expertise in performing and interpreting the examination [23, 24]. In fact, we simplified the complicated procedures of US adopted in the diagnostic examination which need 
Table 2 The functional evaluation of the elbows (affected elbows vs. unaffected elbows)

\begin{tabular}{lll}
\hline Outcomes & Degrees $\left(^{\circ}\right)$ & $\boldsymbol{F}$ \\
\hline Flexion of the affected elbows & $145.26 \pm 4.94(136.0-157.0)$ & 5.154 \\
Flexion of the unaffected elbows & $148.33 \pm 5.01(139.0-161.0)$ & \\
Extension of the affected elbows & $6.63 \pm 4.83(-1.5-14.0)$ & 2.642 \\
Extension of the unaffected elbows & $4.57 \pm 4.45(-6.5-10.0)$ & 0.110 \\
ROM of the affected elbows & $138.63 \pm 5.95(127.0-150.0)$ & 10.045 \\
ROM of the unaffected elbows & $143.76 \pm 5.95(130.5-155.0)$ & 0.003 \\
\hline
\end{tabular}

multi-plane scanning [15], as the preoperative imaging examination has shown the displacements of the fracture comprehensively. As a result, the aim of intraoperative US is to offer the images to assure the reduction of fracture displacements in the lateral and posteroanterior planes. In the present study, the US in the lateral and posterior plane could adequately and effectively show the structures at fracture site and guide intraoperative reduction.

The cubitus varus deformity seems to be the most common complication after TFDH. Nevertheless, the definite reason of the cubitus varus has not been elucidated. Previous studies reported variable incidences of cubitus varus after treatment of TFDH which ranged from 25 to $70 \%$ [25]. In the present series, the incidence of cubitus varus after closed reduction was $7.4 \%$, which is comparable to Hariharan et al.'s recent multicenter review [24]. The lower incidence of the cubitus varus in the present study might be attributed to the following reasons. It has been reported that there is a higher frequency of varus deformity in children less than 2 years of age in the previous studies [26]. However, most patients in the present study were relatively older toddlers with an average age of 15 months which was different from most studies mainly including neonates. Secondly, thanks to the less remodeling potential of the distal humerus, the cubitus varus seems to be not progressive [27]. An inadequate reduction could be the main cause of cubitus varus in toddlers with TFDH [6]. In the present study, all the reduction was guided by the US, and approximately anatomy reduction could be guaranteed in most cases which contributed to the avoidance of the cubitus varus effectively. Thirdly, it has been reported that stable fixation after reduction is also an important factor in preventing cubitus varus [24, 28]. In the present study, considering the maintenance of displaced fracture reduction may be difficult, especially if elbow swelling was presented and then decreased, and plaster immobilization alone could not maintain the reduction stably. All the patients underwent reduction followed by percutaneous pinning fixation. In agreement with previous studies, we deem that this stable fixation procedure was conducive to maintain the position after reduction and further accelerate the callus forming at the fracture site.

There are some limitations in the present study; this is a retrospective study and the follow-up is relatively short; thus, the long-term follow-up of clinical and radiographic outcomes are required to evaluate the development of the cubitus varus. The US could guide the fracture reduction intraoperatively; however, the fixation K-wires were penetrated into the bone

Table 3 Functional and cosmetic outcomes according to Flynn's criteria (affected elbows vs. unaffected elbows)

\begin{tabular}{|c|c|c|c|c|}
\hline \multirow[t]{2}{*}{ Outcomes } & & \multicolumn{2}{|l|}{ Number } & \multirow[t]{2}{*}{$x$} \\
\hline & & Affected elbows & Unaffected elbows & \\
\hline \multirow[t]{4}{*}{ Functional, loss of range of motion (degrees) } & Excellent $(0-5)$ & $21(77.78 \%)$ & $27(100.00 \%)$ & \multirow[t]{4}{*}{0.030} \\
\hline & Good (5-10) & $6(22.22 \%)$ & $0(0.00 \%)$ & \\
\hline & Fair (10-15) & $0(0.00 \%)$ & $0(0.00 \%)$ & \\
\hline & Poor (> 15) & $0(0.00 \%)$ & $0(0.00 \%)$ & \\
\hline \multirow[t]{4}{*}{ Cosmetic, difference in carrying angle (degrees) } & Excellent (0-5) & $23(85.19 \%)$ & $27(100.00 \%)$ & \multirow[t]{4}{*}{0.119} \\
\hline & Good (5-10) & $4(14.81 \%)$ & $0(0.00 \%)$ & \\
\hline & Fair (10-15) & $0(0.00 \%)$ & $0(0.00 \%)$ & \\
\hline & Poor (> 15) & $0(0.00 \%)$ & $0(0.00 \%)$ & \\
\hline
\end{tabular}


Table 4 The radiographic carrying angles (affected elbows vs. unaffected elbows)

\begin{tabular}{lllll}
\hline & $\begin{array}{l}\text { Affected } \\
\text { elbows }\left(^{(}\right)\end{array}$ & $\begin{array}{l}\text { Unaffected } \\
\text { elbows }\left(^{(}\right)\end{array}$ & $\boldsymbol{F}$ & $\boldsymbol{P}$ \\
\hline $\begin{array}{l}\text { Carrying angle of } \\
\text { physical examination }\end{array}$ & $11.37 \pm 4.14$ & $10.93 \pm 2.93$ & 0.027 & 0.651 \\
$\begin{array}{l}\text { Carrying angle of } \\
\text { radiographs }\end{array}$ & $10.46 \pm-19.0)$ & $(2.0-15.0)$ & & \\
\hline
\end{tabular}

which are uneasily delineated by ultrasound. Therefore, radiography is still needed to confirm the configuration of the fixation pins intraoperatively.

In conclusion, our study shows the satisfactory clinical and radiographic outcomes in children with displaced TFDH treated by US-guided closed reduction with percutaneous pining fixation. With the guidance of intraoperative US, the acceptable fracture reduction can be achieved with the advantages of real-time, nonradioactive, and simple utilization.

\section{Authors' contributions}

$\mathrm{YZ}, \mathrm{HZ}, \mathrm{ML}$, and $\mathrm{GZ}$ were involved in the conception and design of the project. All authors participated in the surgery implementation. YZ and GZ collected and extracted the data. $\mathrm{HZ}, \mathrm{YZ}$, and GZ conducted the analysis and data interpretation. $\mathrm{HZ}$ and $\mathrm{YZ}$ drafted the manuscript. $\mathrm{YZ}, \mathrm{HZ}$, and $\mathrm{ML}$ made the critical revisions. All authors read, provided feedback, and approved the final manuscript.

\section{Funding}

This work was supported by the Projects of Chongqing Science and Technology Committee Foundation (cstc2019jcyj-msxmX0853).

\section{Availability of data and materials}

The datasets used and/or analyzed during the current study are available from the corresponding author on reasonable request.

\section{Ethics approval and consent to participate}

The study has received institutional review board approval by the Children's Hospital of Chongqing Medical University (No.2020196).

\section{Consent for publication}

Not applicable.

\section{Competing interests}

The authors declare that they have no competing interests.

\footnotetext{
Author details

'Department of Orthopaedics, Children's Hospital of Chongqing Medical University, 136 Zhongshan Er Road, Yuzhong District, Chongqing 400014, People's Republic of China. ${ }^{2}$ Ministry of Education Key Laboratory of Child, Children's Hospital of Chongqing Medical University, 136 Zhongshan Er Road, Yuzhong District, Chongqing 400014, People's Republic of China. ${ }^{3}$ Development and Disorders, Children's Hospital of Chongqing Medical University, 136 Zhongshan Er Road, Yuzhong District, Chongqing 400014, People's Republic of China. ${ }^{4}$ National Clinical Research Center for Child Health and Disorders, Children's Hospital of Chongqing Medical University, 136 Zhongshan Er Road, Yuzhong District, Chongqing 400014, People's Republic of China. ${ }^{5}$ China International Science and Technology Cooperation base of Child development and Critical Disorders, Children's Hospital of Chongqing Medical University, 136 Zhongshan Er Road, Yuzhong District, Chongqing 400014, People's Republic of China.
}

Received: 5 October 2020 Accepted: 24 November 2020

Published online: 01 December 2020

\section{References}

1. Abzug Joshua M, Ho Christine A, Ritzman Todd F, et al. Transphyseal fracture of the distal humerus. J Am Acad Orthop Surg. 2016;24:e39-44.

2. Sawant MR, Narayanan S, O'Neill K, et al. Distal humeral epiphysis fracture separation in neonates_diagnosis using MRI scan. Injury. 2002;33:179-81.

3. María N, Fina D-L, Jesús A, et al. Distal humeral epiphysiolysis in the newborn: utility of sonography and differential diagnosis. Clin Imaging. 2013:37(1):180-4

4. Matthew K, Carly S, Peter S, et al. Diagnosing neonatal transphyseal fractures of the distal humerus. J Med Imaging Radiat Oncol. 2017:61(4): 494-9. https://doi.org/10.1111/1754-9485.12607.

5. Min CS, Dae SH, Seok CE. Long-term outcomes of distal humeral epiphyseal separations treated via closed reduction and pinning under arthrogram. Injury. 2020;51(2):207-11.

6. Zhou W, Canavese F, Zhang $L$, et al. Functional outcome of the elbow in toddlers with transphyseal fracture of the distal humerus treated surgically. $J$ Child Orthop. 2019:13(1):47-56.

7. Yoo Cl, Suh JT, Suh KT, et al. Avascular necrosis after fracture-separation of the distal end of the humerus in children. Orthopedics. 1992;15(8):959-63.

8. Reza O, Choi Paul D, Skaggs David L. Supracondylar humeral fractures in children. J Bone Joint Surg Am. 2008;90(5):1121-32.

9. Jacobsen S, Hansson G, Nathorst-Westfelt J. Traumatic separation of the distal epiphysis of the humerus sustained at Barth. J Bone Joint Surg Br. 2009:91:797-802.

10. Barrett WP, Almquist EA, Staheli LT. Fracture separation of the distal humeral physis in the newborn. J Pediatr Orthop. 1984;4:617-9.

11. Greg C, Jeannette $K$, Kate $K$. The role of ultrasound as a problem-solving tool in the assessment of paediatric musculoskeletal injuries. Ultrasound. 2019;27(1):6-19.

12. Patil Mallanagouda N. Palled Eranna. Epihyseal separation of lower end humerus in a neonate-diagnostic and management difficulty. J Orthop Case Rep. 2015;5(4):7-9.

13. Wang, P. H, Chern, T. C, Su, W. R, et al. Ultrasonography applied in guiding the reduction and assessing the healing of distal humeral epiphysis fracture-separation in a neonate-a case report. European Journal of Radiology Extra. 2009; 72(2).

14. Chapleau J, Canet F, Petit Y, et al. Validity of goniometric elbow measurements: comparative study with a radiographic method. Clin Orthop Relat Res. 2011:469(II):3134-40.

15. Kolja E, Ole A, Bernd S, et al. Ultrasound evaluation of elbow fractures in children. J Med Ultrason (2001). 2013:40(4):443-51.

16. Nucharin S, Hicks Ralph A, Beth CC, et al. Distal humeral epiphyseal separation in young children: an often-missed fracture-radiographic signs and ultrasound confirmatory diagnosis. AJR Am J Roentgenol. 2015;204(2): W192-8.

17. Dias JJ, Lamont AC, Jones JM. Ultrasonic diagnosis of neonatal separation of the distal humeral epiphysis. J Bone Joint Surg Br. 1988;70(5):825-8.

18. Ziv N, Litwin A, Katz K, et al. Definitive diagnosis of fracture-separation of the distal humeral epiphysis in neonates by ultrasonography. Pediatr Radiol. 1996;26(7):493-6.

19. Grechenig W, Clement HG, Fellinger $M$, et al. Scope and limitations of ultrasonography in the documentation of fractures--an experimental study. Arch Orthop Trauma Surg. 1998;117:368-71.

20. Lei C, Yunie K, Moore Christopher L. Diagnosis and guided reduction of forearm fractures in children using bedside ultrasound. Pediatr Emerg Care. 2007:23(8):528-31.

21. Wellsh Bobby M, Kuzma JM. Ultrasound-guided pediatric forearm fracture reductions in a resource-limited ED. Am J Emerg Med. 2016;34(1):40-4.

22. Zhao Z, Zhu J, Song F, et al. Intraoperative ultrasound-guided reduction of femoral shaft fractures using intramedullary nailing: a technical note. Arch Orthop Trauma Surg. 2019;139(5):589-96.

23. Beckmann NM, Crawford L. Salter-Harris I fracture of the distal humerus in a neonate: imaging appearance of radiographs, ultrasound, and arthrography. Radiology Case Reports. 2017;19:780.

24. Hariharan A R, Ho C, Bauer A, et al. Transphyseal humeral separations: what can we learn? A retrospective, multicenter review of surgically treated patients over a 25-year period. Journal of Pediatric Orthopaedics. 2019; publish ahead of print 
25. Tudisco Cosimo, Mancini Federico, De Maio Fernando, et al. Fractureseparation of the distal humeral epiphysis. Long-term follow-up of five cases. Injury. 2006; 37(9): 843-848.

26. de Jager LT, Hoffman EB. Fracture-separation of the distal humeral epiphysis. J Bone Joint Surg Br. 1991;73(1):143-6.

27. Dimeglio A. Growth in pediatric orthopaedics. J Pediatr Orthop. 2001;21(4): 549-55.

28. Abe M, Ishizu T, Nagaoka T, et al. Epiphyseal separation of the distal end of the humeral epiphysis: a follow-up note. J Pediatr Orthop. 1995;15:426-34.

\section{Publisher's Note}

Springer Nature remains neutral with regard to jurisdictional claims in published maps and institutional affiliations.

Ready to submit your research? Choose BMC and benefit from:

- fast, convenient online submission

- thorough peer review by experienced researchers in your field

- rapid publication on acceptance

- support for research data, including large and complex data types

- gold Open Access which fosters wider collaboration and increased citations

- maximum visibility for your research: over $100 \mathrm{M}$ website views per year

At $B M C$, research is always in progress.

Learn more biomedcentral.com/submissions 